\title{
Investigation of thermohydraulic dispatcher properties and peculiarities
}

\author{
D. O. Romanov ${ }^{1, *}$, and $Y . V$. Yavorovsky ${ }^{1}$ \\ ${ }^{1}$ National Research University "Moscow Power Engineering Institute", Moscow, Russia
}

\begin{abstract}
Application of thermohydraulic dispatcher (THD) for district heating systems is, to some extent, limited due to insufficient information about its properties and features. At the same time this device has a potential to solve the problem of mutual dependence of heat consumers and to decrease electricity consumption in heat supply systems. This paper deals with experimental research and numerical simulation of THD with the aim of increasing the range of knowledge about its properties. Presented results describe already known properties such as near-to-zero differential pressure at THD and flow rates independence which were checked. Some new properties and peculiarities of THD were obtained during the investigation as well. Among them is differential pressure "variability", hydrodynamic separation under higher velocity, similarity of thermohydraulic processes inside THD under similar velocities in manifolds of THD, absence of thermal stratification in mixing regime, dependence of secondary circuits supply temperature from the height level and secondary circuits return temperature.
\end{abstract}

\section{Introduction}

Thermohydraulic dispatcher (THD) is vertical or horizontal pipe manifold of large diameter which has low hydraulic resistance [1]. Although THD is quite often used in local decentralised heat supply systems [2], there is not enough information about its properties backed by theoretical and experimental research. It is important to know more about THD properties in order to use it rationally in centralized heat supply systems. Existing mathematical models of thermohydraulic dispatcher describe flow rates and pressure drops [3-4]. Other mathematical models were developed to describe operating regimes of THD [5]. But these models do not describe heat and hydraulic processes inside the device. So, experimental research and numerical simulation of thermohydraulic dispatcher were conducted to get better understanding of THD properties and peculiarities. The initial stage of the research was published in [6].

\section{Methods}

\subsection{Experimental research}

Experimental investigation of THD properties was conducted by means of the experimental installation shown in Figure 1. Temperatures, pressures $(4-20 \mathrm{~mA}$ signal), differential pressures (4-20 mA signal), volume flow rates (visual indication) were measured during experiments. Randomized layout of the experiment was applied [7]. Thermal images were obtained by means of thermal camera.

\subsection{Numerical simulation}

In order to further investigate internal properties of THD, simulation model was created in ANSYS Fluent software. The geometrical model of THD is symmetrical and the fields of parameters change symmetrically, so only half of the geometrical model is needed. Therefore, flow rates were divided by two for correct simulation. The process of creating computational mesh is semiautomatic with program controlled inflation. Minimum orthogonal quality of mesh is 0.26 and maximum skewness is 0.75 [8]. Experimental data was used as boundary conditions. Input boundary conditions were mass flow rate and temperature. Output boundary condition was outflow. Three models of turbulence were tested: k- $\varepsilon$ Standard, k- $\varepsilon$ Realizable, k- $\omega$ SST [9-11]. Among them k- $\varepsilon$ Standard model showed more accurate results, when comparing to experimental results and thermal images, and it was used for further simulations.

\section{Results}

Figure 2 shows dependence of pressure drop at THD manifolds from volume flow rate. This experiment was conducted when each circuit operated separately, one at a time. It can be seen that pressure drop at THD manifolds is relatively small and the values are almost the same for all 3 circuits.

\footnotetext{
* Corresponding author: dimrom09@gmail.com
} 


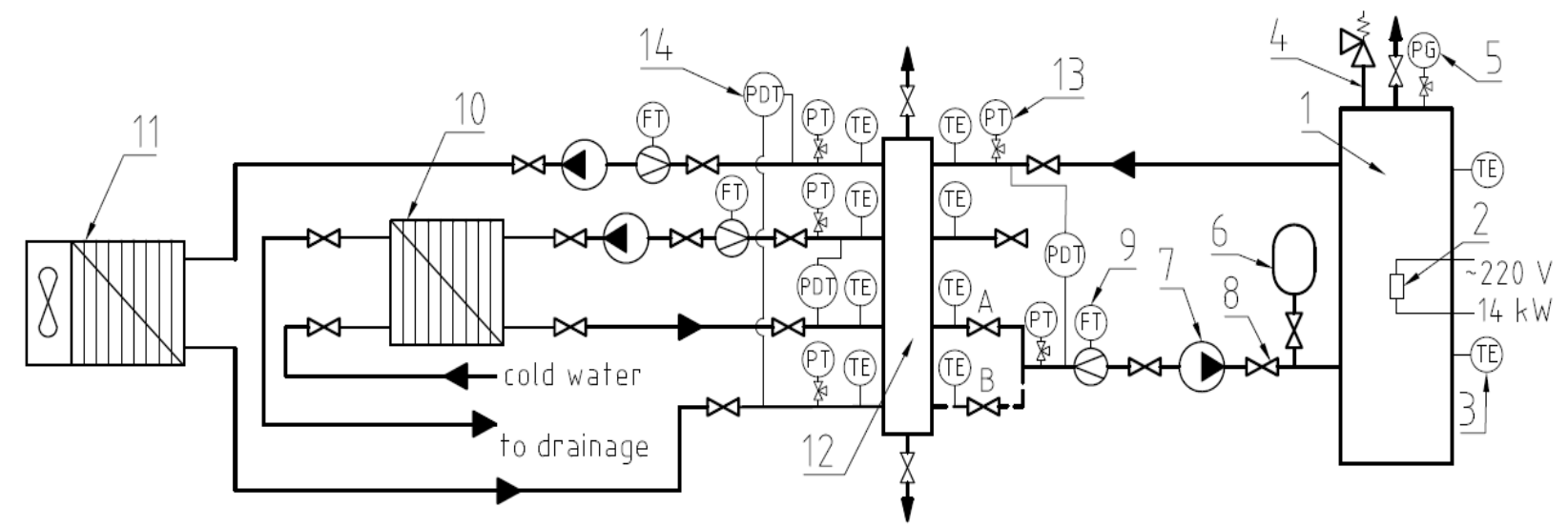

Fig. 1. The principal scheme of the experimental installation. 1 - boiler (primary circuit "I"), 2 - electric heaters, 3 - resistance thermometer, 4 - safety valve, 5 - manometer, 6 - expansion vessel, 7 - pump, 8 - shut-off valve, 9 - electromagnetic flow meter, 10 - plate heat exchanger (secondary circuit "ex"), 11 - calorifer (secondary circuit "cal"), 12 - thermohydraulic dispatcher, 13 - pressure sensor, 14 - differential pressure sensor, A - connection for non-condensation boilers, B - connection for condensation boilers.

Configurations for connecting condensation and noncondensation ("\#”) boilers also gave the same results during the experiment. Moreover, increasing temperature of fluid up to $70^{\circ} \mathrm{C}$ had no effect on hydraulic regime of THD. Since sensors were installed at manifolds, they could not measure pressure drop at THD directly. This value, estimated by numerical simulation, is less than $100 \mathrm{~Pa}$. Therefore, experimentally measured values represent, mainly, friction and local pressure losses in pipe manifolds.

Figure 3 shows dependence of mass flow rate relative change in primary circuit "I" from mass flow rate in secondary circuit "ex". While mass flow rate in "ex" circuit changes from 100 to $1000 \mathrm{~kg} / \mathrm{hr}$, relative change in "I" circuit is not more than $1 \%$. Similar results were obtained for other circuits. So, mass flow rate in circuits do not affect each other and hydrodynamic separation of circuits can be achieved. It is worth noting that some researchers advise to maintain the fluid velocity in THD less than $0.1-0.2 \mathrm{~m} / \mathrm{s}$ [12-13]. But in these experiments the velocity reached up to $0.25 \mathrm{~m} / \mathrm{s}$ and the circuits still did not affect each other. So, hydrodynamic separation was observed under higher velocity than recommended.

Figure 4 shows dependence of pressure drop at THD manifolds ("I" circuit) from mass flow rate in "I" circuit. In contrast to Figure 2, this dependence was obtained when two circuits ("I" and "ex") operated simultaneously. It can be seen that several values of differential pressure correspond to single value of mass flow rate in "I" circuit. The more the flow rate in "ex" circuit, the less differential pressure at THD manifolds ("I" circuit). If there is no flow rate in "ex" circuit, then differential pressures in "I" circuit are equal to values from Figure 2. Similar effect of differential pressure "variability" was noticed for "cal" circuit. Numerical simulation also proved this peculiarity. It is observed when manifolds of primary and secondary circuits are located on the same height level facing each other (upper

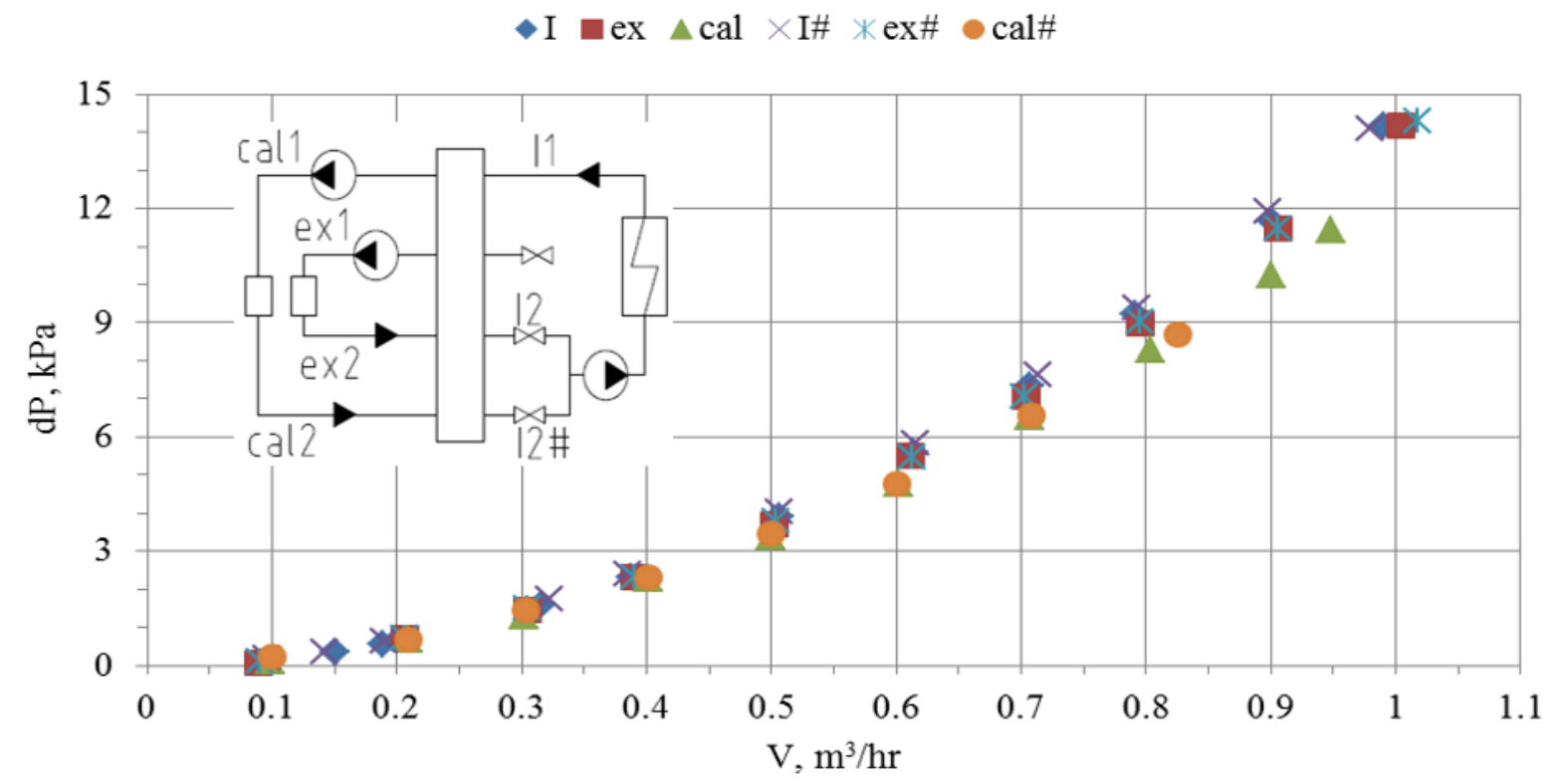

Fig. 2. Dependence of pressure drop at THD manifolds from volume flow rate. 


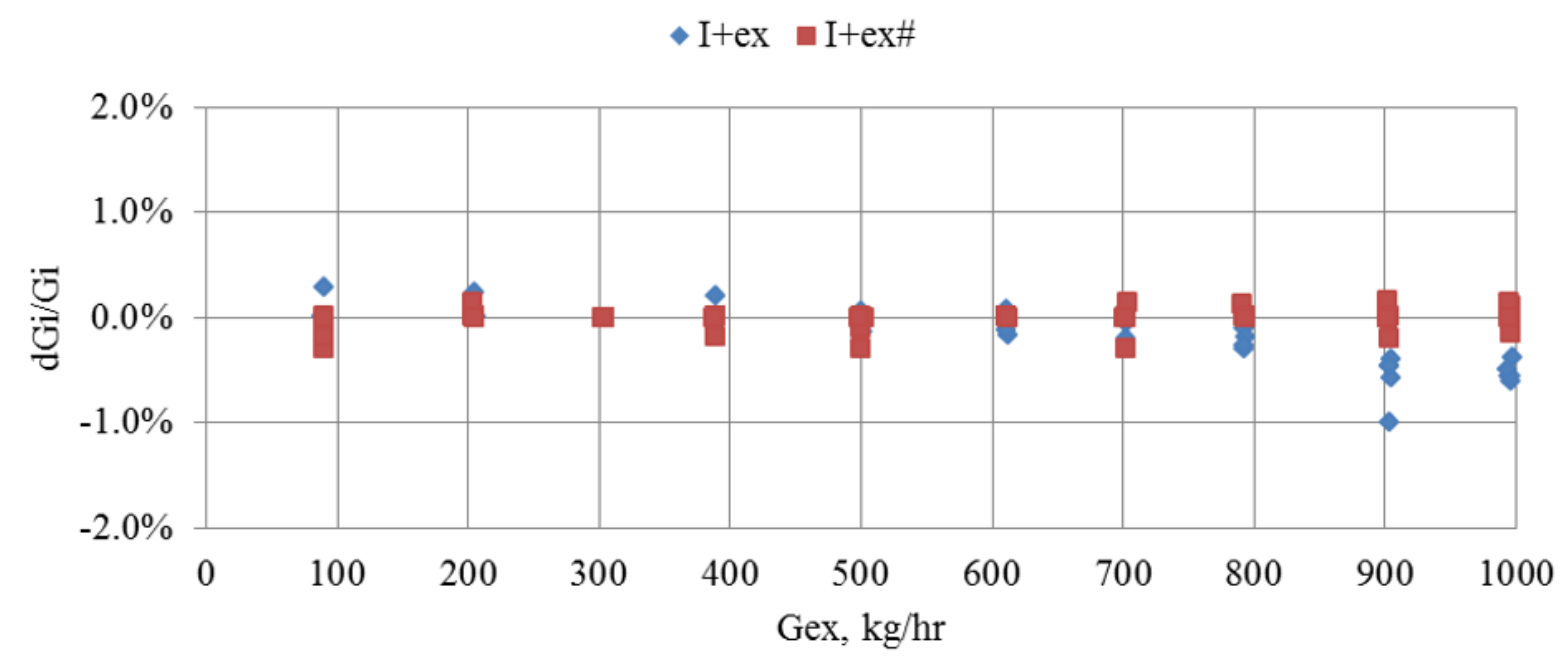

Fig. 3. Dependence of mass flow rate relative change in primary circuit ("I") from mass flow rate in secondary circuit ("ex").

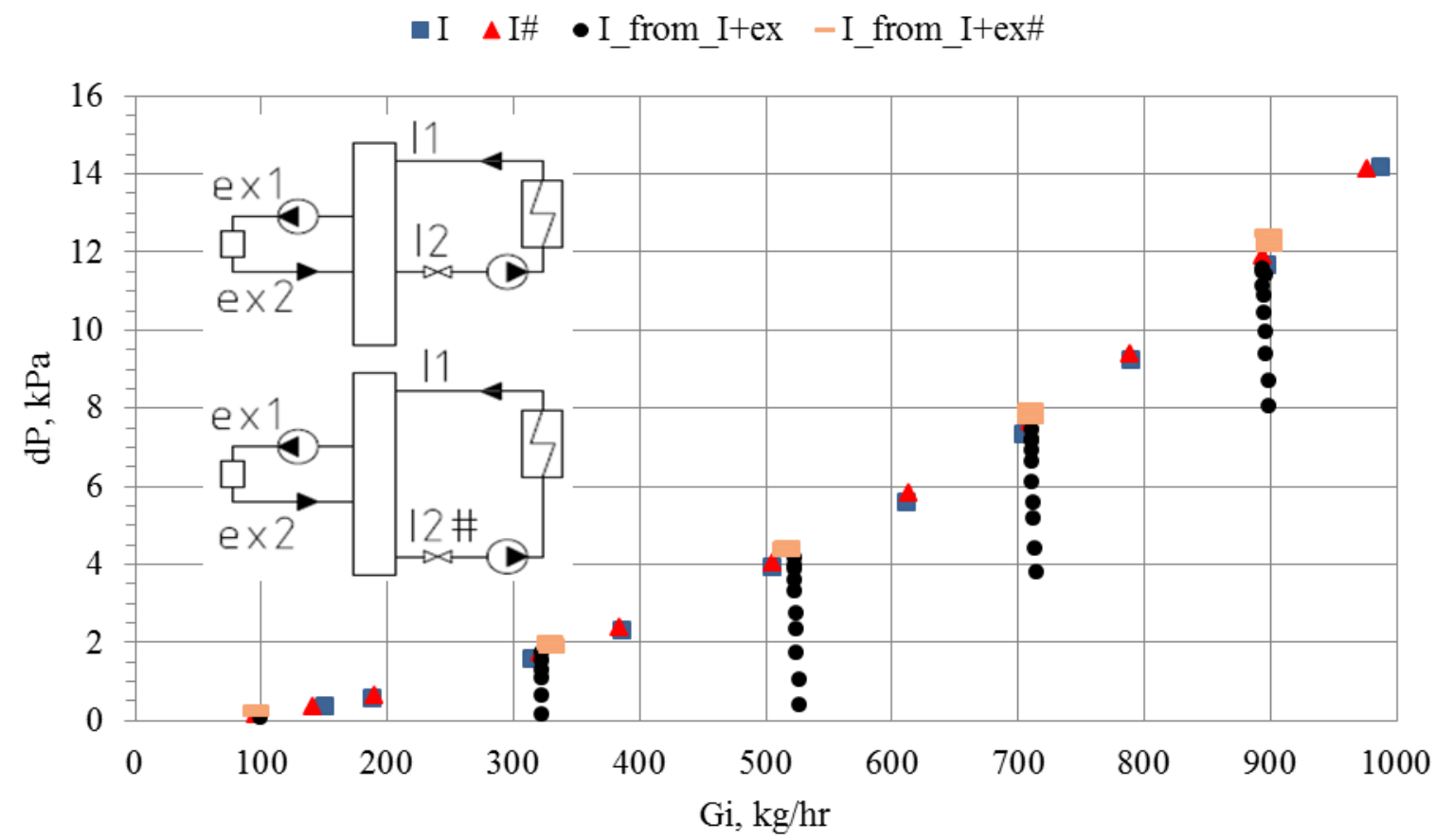

Fig. 4. Dependence of pressure drop at THD manifolds ("I") from mass flow rate in "I" circuit.

scheme in Figure 4). When manifolds are on the same level, liquid easier comes from one manifold to the manifold across. In this case, the interconnection between flows exists and it makes differential pressure change due to conversion of dynamic pressure into static pressure according to Bernoulli's principle. When manifolds are on different height levels, liquid knocks the wall and loses a part of its kinetic energy. Experimental results showed absence of differential pressure "variability" when manifolds are not on the same height level. So, it can be recommended to locate primary and secondary manifolds on different levels (lower scheme in Figure 4) while constructing THD in order to avoid the effect of differential pressure "variability". This effect might cause delusion of control systems and operating personnel.
Maximum absolute errors of the experiments were calculated according to [14-15] and shown in Table 1.

Table 1. Maximum absolute errors of the measurements.

\begin{tabular}{|c|c|c|}
\hline Maximum absolute error & Unit & Value \\
\hline Absolute pressure & $\mathrm{kPa}$ & 5 \\
\hline Differential pressure & $\mathrm{kPa}$ & 0.5 \\
\hline Temperature & ${ }^{\circ} \mathrm{C}$ & 0.7 \\
\hline Volume flow rate & $\mathrm{m}^{3} / \mathrm{hr}$ & 0.004 \\
\hline
\end{tabular}

The numerical model with $\mathrm{k}-\varepsilon$ Standard turbulence model was used to investigate other THD properties. 
Several numerical simulations were conducted and it turned out that increasing linear and radial dimensions of initial numerical model by 5 fold led to increasing flow rates by 25 fold in order to get the same temperature field inside THD and in its manifolds as for initial model. So, similarity of thermohydraulic processes inside THD is reached under similar liquid velocities in manifolds;

Thermal stratification along the height of THD is not confirmed for mixing regime (when total flow rate in primary circuits is less than that in secondary circuits). Figure 5 shows thermal images of upper and lower parts of THD in mixing regime and Figure 6 shows temperature field on external surface of THD as a result of mixing regime simulation. It can be seen on both figures that THD has warm layers of liquid at the top and bottom, while the center contains cold layer.

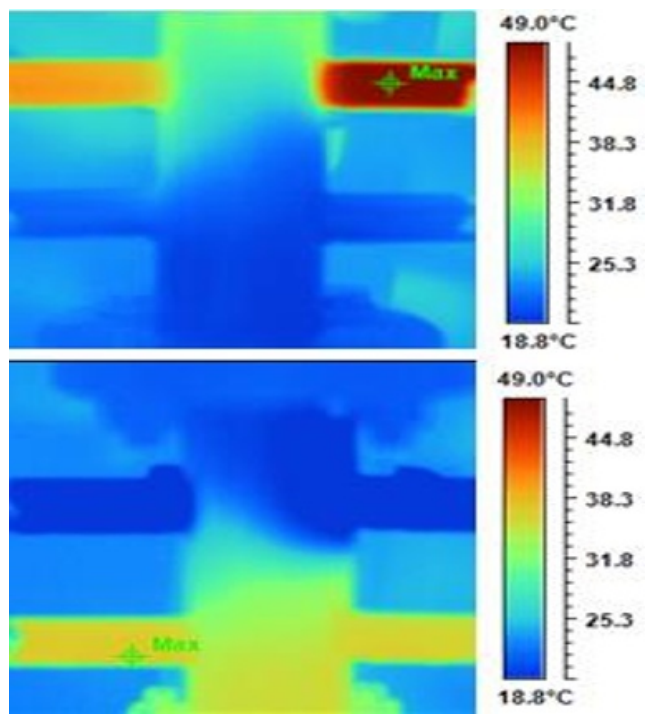

Fig. 5. Thermal images of upper and lower parts of THD in mixing regime.

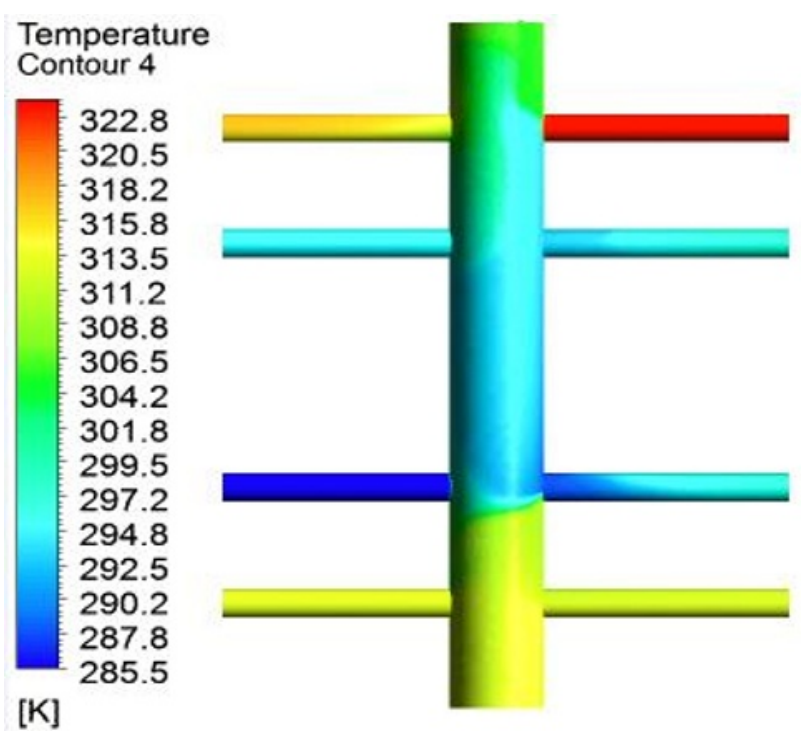

Fig. 6. Temperature field on external surface of THD as a result of mixing regime simulation.

Initial numerical model with 4 manifolds was transformed into model with 8 manifolds (4 supply manifolds and 4 return manifolds). Simulation was made under equal total flow rates in primary and secondary circuits (nominal regime). Figure 7 shows dependence of temperature in supply manifolds of secondary circuits from temperature in return manifolds of secondary circuits and from the height level of manifolds. The higher the number at $\mathrm{Y}$-axis, the higher a manifold is located. In result, temperatures in supply manifolds № 4, 3,2 of secondary circuits deviate from supply temperature of primary circuit by $2-3{ }^{\circ} \mathrm{C}$. At the same time deviation of temperature in supply manifolds № 1 is 3-5 ${ }^{\circ} \mathrm{C}$. This might be caused by proximity of return manifolds to supply manifolds № 1 . Moreover, the lower return temperature, the lower supply temperature of secondary circuits. The least temperature deviation was observed in the upper supply manifold (№ 4).

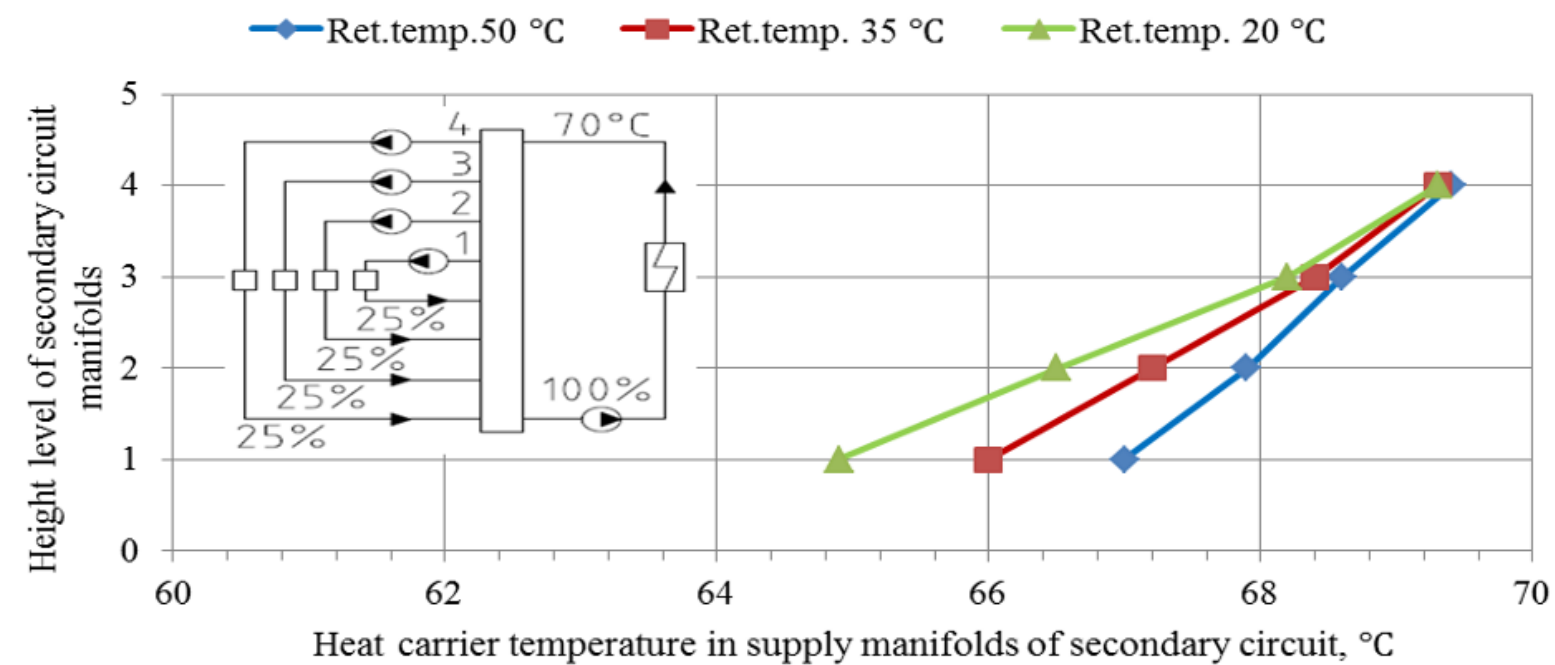

Fig. 7. Dependence of temperature in supply manifolds of secondary circuits from temperature in return manifolds of secondary circuits and from the height level of manifolds in nominal regime. 


\section{Discussion}

The investigated properties of THD can be used for its correct construction and designing heat supply systems with THD. But there could be other aspects worth investigating such as optimal geometrical configuration and dimensions of THD. This topic is especially important when it comes to designing large centralised district heating systems with THD. In this case, THD may have large dimensions and massive construction. So, defining the extent to which it is reasonable to create THD of conventional design is the question of significant importance. Similar to this subject is the question of optimal amount of THD manifolds taking into account that it is better to locate manifolds on different height levels and thus, increasing overall THD height. The results of this paper have shown that return manifolds affect temperature in supply manifolds of secondary circuits. Then the question is whether return manifolds should be located further from supply manifolds and thus, again increasing overall THD height. All of the above mentioned questions are subjects of feasibility study, since, on the one hand, they deal with technical improvements or restrictions of THD construction but, on the other hand, they require increasing dimensions of THD and corresponding increase of materials, labour work, and other producing costs.

\section{Conclusion}

In result of experimental research and numerical simulation, already known properties of THD such as near-to-zero differential pressure at THD and flow rates independence were checked and new properties and peculiarities of THD were found. Among them is differential pressure "variability", hydrodynamic separation under higher velocity, similarity of thermohydraulic processes inside THD under similar velocities in manifolds of THD, absence of thermal stratification in mixing regime, dependence of secondary circuits supply temperature from the height level and secondary circuits return temperature.

The work was performed under support of the state order № 13.9613.2017/8.9.

\section{References}

[1] Y.V. Yavorovsky, D.O. Romanov, et al. Application of thermohydraulic dispatcher in low temperature district heating systems for decreasing heat carrier transportation energy cost and increasing reliability of heat supply IOP Conf. Series: Journal of Physics: Conf. Series 891 (2017)

[2] P.A. Khavanov, Heat supply sources of autonomous systems (in Russian) (2014)

[3] V.V. Sennikov, Y.V. Genvarev et al. Application of thermohydraulic dispatcher in district (2009)
[4] V.V. Sennikov, Y.V. Genvarev, et al. Application of thermohydraulic dispatcher in heating substations of centralized heat supply system Vestnik IGEU 4, 15-20 (in Russian) (2012)

[5] D.O. Romanov, Y.V. Yavorovsky, et al. Experimental research of thermohydraulic dispatcher operating regimes Safety and Realiability of Power Industry 2, 106-112 (in Russian) (2019)

[6] Y.V. Yavorovsky, D.O. Romanov, et al. Experimental research of thermo-hydraulic separators and dispatchers in heat supply systems Solid State Phenomena 284, 1385-89 (2018)

[7] H. Schenck, Theories of engineering experimentation (1972)

[8] ANSYS Fluent Meshing Users Guide. 15.0. (Canonsburg, PA: ANSYS, Inc.) (2013)

[9] ANSYS Fluent Theory Guide. 15.0. (Canonsburg, PA: ANSYS, Inc.) (2013)

[10] A.V. Garbaruk, M.K. Strelets, et al. Modern approaches to turbulence modelling (In Russian) (2016)

[11] A.A. Anikeev, A.M. Molchanov, et al. Basics of computational fluid dynamics and heat exchange (In Russian) (2010)

[12] P.A. Khavanov, Autonomous heat supply sources with collectors of low differential pressure AVOK 3, 26-35 (in Russian) (2002)

[13] R. Yauschovets, Hydraulics is the heart of water heating system (in Russian) (2005)

[14] V.V. Kurepin, I.V. Baranov Processing of experimental data (in Russian) (2003)

[15] I.V. Mitin, V.S. Rusakov, Analysis and processing of experimental data (in Russian) (1998) 